\title{
Statistical mechanics of two hard disks in a rectangular box
}

$\operatorname{AUTHOR}(\mathrm{S})$ :

Munakata, T; Hu, G

\section{CITATION:}

Munakata, T ...[et al]. Statistical mechanics of two hard disks in a rectangular box. Physical Review E 2002, 65(6): 066104.

ISSUE DATE:

2002-06

URL:

http://hdl.handle.net/2433/50310

RIGHT:

Copyright 2002 American Physical Society 
PHYSICAL REVIEW E, VOLUME 65, 066104

\title{
Statistical mechanics of two hard disks in a rectangular box
}

\author{
Toyonori Munakata \\ Department of Applied Mathematics and Physics, Kyoto University, Kyoto 606, Japan \\ Gang $\mathrm{Hu}$ \\ Department of Physics, Beijing Normal University, Beijing 100875, People's Republic of China
}

(Received 7 January 2002; published 13 June 2002)

\begin{abstract}
A system of two hard disks in a rectangular box is studied based on the exact partition function and equilibrium distribution functions of particles. Box-size dependence of some quantities of interest, such as pressure and the particle distribution functions, is investigated and in particular the negative compressibility of the van der Waals type and the corresponding phase transition are analyzed in detail. This system turns out to have rich structures that are related to the ergode-nonergode transitions in this system.
\end{abstract}

DOI: 10.1103/PhysRevE.65.066104

PACS number(s): 05.20.Jj, 05.70.Fh, 61.20.Gy

As is well known, chaos and ergodicity $[1,2]$ serve as a bridge linking mechanics and statistics. This bridge is not limited to many-body systems, a main field for application of statistical mechanics. Currently, some few-body systems gather considerable attention in this regard and for few-body hard-core (or disk) systems, entropy [3], the thermodynamic second law [4], and a phase transition [5] are discussed to mention a few.

We consider a system composed of two identical hard disks put in a rectangular box, which was studied by Awazu [5] with a molecular dynamics (MD) method and a liquidsolid-like transition [6] with negative compressibility was observed to exist. It is noted, however, that without explicit analysis, the understanding of the mechanism underlying the interesting behavior is not complete and the purpose of this paper is to study the system by entirely analytical computation and understand the van der Waals features in the large density case statistical mechanically.

We denote the diameter of a hard disk by $d$ and a horizontal (vertical) length of the rectangular box by $l_{x}+d\left(l_{y}\right.$ $+d$ ). It is remarked that as $l_{x}$ is decreased from above $d$ to below $d$, the system naturally shows an ergode-nonergode transition, in which a particle occupying the upper part of the box is kept from going into the lower part when $l_{x}$ becomes smaller than $d$. In order to take into account the particle-wall interaction, we introduce the coordinate system $(x, y)$, in which $x$ and $y$ can take values in the range $0 \leqslant x \leqslant l_{x}$ and 0 $\leqslant y \leqslant l_{y}$, respectively. With use of $(x, y)$, the distribution function of the position coordinates $\left\{x_{i}, y_{i}(i=1,2)\right\}$ is simply given by $p\left(x_{1}, y_{1} ; x_{2}, y_{2}\right)=1 / Z_{c}$ for $R \equiv\left[\left(x_{1}-x_{2}\right)^{2}+\left(y_{1}\right.\right.$ $\left.\left.-y_{2}\right)^{2}\right]^{1 / 2} \geqslant d$ and $p\left(x_{1}, y_{1} ; x_{2}, y_{2}\right)=0$ for $R<d$. Here, the configurational partition function $Z_{c}\left(l_{x}, l_{y}\right)$ normalizes the probability distribution function by

$$
\int d x_{1} d x_{2} d y_{1} d y_{2} p\left(x_{1}, y_{1} ; x_{2}, y_{2}\right)=1
$$

Let us consider the probability distribution functions $f(x)$ and $g(y)$ for the relative coordinates $x=x_{2}-x_{1}$ and $y=y_{2}$ $-y_{1}$, which were numerically obtained before [5]. For example, $f(x)$ is defined by

$$
f(x) \equiv \int d x_{1} d x_{2} d y_{1} d y_{2} p\left(x_{1}, y_{1} ; x_{2}, y_{2}\right) \delta\left(x-\left[x_{2}-x_{1}\right]\right)
$$

It is convenient to divide the space $\left(l_{x}, l_{y}\right)$ into four regions, $l_{x} \geqslant d, l_{y} \geqslant d$ (region I), $l_{x} \geqslant d, l_{y}<d$ (region II), $l_{x}<d, l_{y} \geqslant d$ (region III), and $l_{x}<d, l_{y}<d$ (region IV). Simple symmetry consideration reveals that in the region I and IV, $f(x)$ and $g(y)$ are even and we have

$$
f_{\mathrm{I}}\left(x \mid l_{x}, l_{y}\right)=g_{\mathrm{I}}\left(x \mid l_{y}, l_{x}\right), \quad f_{\mathrm{IV}}\left(x \mid l_{x}, l_{y}\right)=g_{\mathrm{IV}}\left(x \mid l_{y}, l_{x}\right),
$$

where size dependence of the functions $f$ and $g$ is shown explicitly. In regions II, $g(y)$ is even and $f(x)$ is to be treated only for, e.g., $l_{x} \geqslant x \geqslant 0$, since two particles cannot change their left-right relationship in the course of time. Symmetry consideration tells us that

$$
g_{\mathrm{III}}\left(y \mid l_{x}, l_{y}\right)=f_{\mathrm{II}}\left(y \mid l_{y}, l_{x}\right), \quad g_{\mathrm{II}}\left(y \mid l_{x}, l_{y}\right)=f_{\mathrm{III}}\left(y \mid l_{y}, l_{x}\right) .
$$

We start from region I. When $l_{x} \geqslant x \geqslant d$, the contribution to $f(x)$ from $\int d y_{1}$ and $\int d y_{2}$ is $l_{y}^{2}$ and from the relation 0 $\leqslant x_{1} \leqslant l_{x}-x$, the contribution from $x_{1}$ integration is $\left(l_{x}-x\right)$. Thus we have

$$
f(x)=l_{y}^{2}\left(l_{x}-x\right) / Z_{c} \quad\left(d \leqslant x \leqslant l_{x}\right) .
$$

When $0 \leqslant x \leqslant d$ and if we confine the contribution to $f(x)$ from the region $y_{1} \leqslant y_{2}$, we have the factor $l_{y}-\left[y_{1}+\left(d^{2}\right.\right.$ $\left.\left.-x^{2}\right)^{1 / 2}\right]$ from $y_{2}$ integration and this is first integrated from 0 to $l_{y}-\left(d^{2}-x^{2}\right)^{1 / 2}$ over $y_{1}$ and then multiplied by $l_{x}-x$ as the contribution from $x_{1}$ integration. Finally, this is to be multiplied by 2 since the region $y_{1} \geqslant y_{2}$ gives precisely the same contribution as above to $f(x)$. Thus immediately we have

$$
f(x)=\left(l_{x}-x\right)\left[l_{y}-\left(d^{2}-x^{2}\right)^{1 / 2}\right]^{2} / Z_{c} \quad(0 \leqslant x \leqslant d) .
$$

Noting that $f(x)$ is even in $x$, we obtain $Z_{c}$ from the condition $\int_{-l_{x}}^{l_{x}} f(x)=1$ to be 


$$
\begin{aligned}
Z_{c}\left(l_{x}, l_{y}\right) & =l_{y}^{2}\left(l_{x}-d\right)^{2}+2 \int_{0}^{d}\left[l_{y}-\left(d^{2}-y^{2}\right)^{1 / 2}\right]^{2}\left(l_{x}-y\right) d y \\
& =l_{x}^{2} l_{y}^{2}-\pi d^{2} l_{x} l_{y}+(4 / 3)\left(l_{x}+l_{y}\right) d^{3}-d^{4} / 2
\end{aligned}
$$

In region II $f(x)$ is considered only in region $0 \leqslant x \leqslant l_{x}$ as noted before. Note that $x$ cannot be smaller than $x_{\text {min }} \equiv\left(d^{2}\right.$ $\left.-l_{y}^{2}\right)^{1 / 2}$. In the region $d \leqslant x \leqslant l_{x}, f(x)$ is given as before by Eq. (3). When $x_{\min } \leqslant x \leqslant d, f(x)$ is given by Eq. (4), that is,

$$
\begin{gathered}
f(x)=\left(l_{x}-x\right)\left[l_{y}-\left(d^{2}-x^{2}\right)^{1 / 2}\right]^{2} / Z_{c} \quad\left(x_{\min } \leqslant x \leqslant d\right), \\
f(x)=0 \quad\left(0 \leqslant x \leqslant x_{\min }\right) .
\end{gathered}
$$

From the normalization condition $\int_{0}^{l_{x}} f(x) d x=1$, we have

$$
\begin{aligned}
Z_{c}\left(l_{x}, l_{y}\right)= & l_{y}^{2}\left(l_{x}-d\right)^{2} / 2+2 \int_{\sqrt{d^{2}-l_{y}^{2}}}^{d}\left[l_{y}-\left(d^{2}-y^{2}\right)^{1 / 2}\right]^{2} \\
& \times\left(l_{x}-y\right) d y \\
= & l_{x}^{2} l_{y}^{2} / 2-l_{y}^{4} / 12+2 l_{x} d^{3} / 3+l_{y}^{2} d^{2} / 2-l_{x} l_{y} d^{2} \theta-l_{x} \\
& \times\left(l_{y}^{2}+2 d^{2}\right)\left(d^{2}-l_{y}^{2}\right)^{1 / 2} / 3,
\end{aligned}
$$

where $\sin \theta=l_{y} / d$. As $l_{y}$ approaches $d$ from below $d$ in Eq. (8) and from above $d$ in Eq. (5), we notice that $Z_{c}\left(l_{x}, l_{y} \uparrow d\right)=Z_{c}\left(l_{x}, l_{y} \downarrow d\right) / 2$, reflecting the nonergodeergode transition at $l_{y}=d$. As to the probability distribution function of $y \equiv y_{2}-y_{1}$, it is easily obtained that

$$
g(y)=\left(l_{y}-|y|\right)\left[l_{x}-\left(d^{2}-y^{2}\right)^{1 / 2}\right]^{2} /\left(2 Z_{c}\right) \quad\left(0 \leqslant|y| \leqslant l_{y}\right) .
$$

The results for region III are easily obtained from Eq. (2).

Finally, let us consider region IV. If $l_{x}^{2}+l_{y}^{2} \leqslant d^{2}$, the box can contain only one particle. For $l_{x}^{2}+l_{y}^{2}>d^{2}$, we have four ergodic components (if we regard two particles distinguishable), in each of which two particles are distributed along one of two diagonal lines of the box. The distribution function $f(x)$ of the relative coordinate $x_{2}-x_{1}(>0)$ is given by

$$
\begin{gathered}
f(x)=\left(l_{x}-x\right)\left[l_{y}-\left(d^{2}-x^{2}\right)^{1 / 2}\right]^{2} /\left(2 Z_{c}\right) \\
{\left[\left(d^{2}-l_{y}^{2}\right)^{1 / 2} \leqslant x \leqslant l_{x}\right],} \\
f(x)=0 \quad\left[0 \leqslant x \leqslant\left(d^{2}-l_{y}^{2}\right)^{1 / 2}\right] .
\end{gathered}
$$

$Z_{c}$ is calculated from $\int_{0}^{l_{x}} f(x)=1$ to have

$$
\begin{aligned}
Z_{c}\left(l_{x}, l_{y}\right)= & (1 / 2) \int_{\sqrt{d^{2}-l_{y}^{2}}}^{l_{x}}\left[l_{y}-\left(d^{2}-y^{2}\right)^{1 / 2}\right]^{2}\left(l_{x}-y\right) d y \\
= & l_{x}^{2} l_{y}^{2} / 4-\left(l_{x}^{4}+l_{y}^{4}\right) / 24+\left(l_{x}^{2}+l_{y}^{2}\right) d^{2} / 4 \\
& +l_{x} l_{y} d^{2}\left(\pi / 2-\theta_{1}-\theta_{2}\right) / 2 \\
& -\left(d^{2}-l_{y}^{2}\right)^{1 / 2}\left[d^{2} l_{x} / 3+l_{x} l_{y}^{2} / 6\right] \\
& -\left(d^{2}-l_{x}^{2}\right)^{1 / 2}\left[d^{2} l_{y} / 3+l_{y} l_{x}^{2} / 6\right]+d^{4} / 8,
\end{aligned}
$$

where $\sin \theta_{1}=l_{x} / d$ and $\sin \theta_{2}=l_{y} / d$. It is easily confirmed that as $l_{x}$ goes to $d$ in Eq. (8); the $Z_{c}$ is twice the $Z_{c}$ obtained from Eq. (12) in the same limit. This is due to the ergodenonergode transition additional to the one already mentioned just below in Eq. (8). It is noted, however, that there is no discontinuity in pressure, defined below by Eq. (14), as is readily seen from $Z_{c}$ expressed by integral forms in Eqs. (5), (8), and (12).

Now we turn to the equation of state of our system. The configurational part of the entropy $S_{c}$ is given by

$$
S_{c}\left(l_{x}, l_{y}\right)=k_{B} \ln Z_{c}\left(l_{x}, l_{y}\right),
$$

where $k_{B}$ denotes the Boltzmann constant. Pressure on the wall is expressed by

$$
p_{x}\left(l_{x}, l_{y}\right)=T \partial S_{c} / \partial l_{x}, \quad p_{y}\left(l_{x}, l_{y}\right)=T \partial S_{c} / \partial l_{y},
$$

with $T$ the temperature of the system. For hard-disk systems $T$ determines only a time scale and hereafter we put $k_{B}=1$, and $T=1$ for simplicity. It is remarked that our system is thermodynamically characterized by $\left(l_{x}, l_{y}\right)$ and from symmetry $p_{x}\left(l_{x}, l_{y}\right)=p_{y}\left(l_{y}, l_{x}\right)$.

The unisotropic pressure obtained by MD experiments [5] in some part of $\left(l_{x}, l_{y}\right)$ region $\left(l_{y}>d\right)$ was confirmed to be consistent with our analytic results of Eq. (14). As examples, some $p_{x}$ for $l_{y}>d$ are shown in Fig. 1 as a function of $l_{x}$. We notice that the pressure $p_{x}$ on the side (left or right) walls shows the van der Waals characteristics for $d<l_{y}<l_{y, c}$ where $l_{y, c}(=2.106 d)$ denotes the (upper) critical value of $l_{y}$ to be discussed later. In contrast, $p_{y}$ only monotonically decreases with $l_{x}$ for $l_{y}>d$.

Usually the van der Waals behavior is ascribed to an interplay between the short-range repulsion and the long-range attraction in the interatomic potential [7]. The long-range attraction tends to reduce the pressure on the wall by means of a slight density increase inside the container of the gas. The hard-disk system shown in Fig. 1 is highly compressed and inhomogeneous. In Fig. 2, we plot, for $l_{y} / d=1.2$, the probability distribution function of $x$ coordinate of a particle $n_{x}(x)$, defined by

$$
n_{x}(x) \equiv \int d x_{1} d y_{1} d y_{2} p\left(x_{1}, y_{1} ; x, y_{2}\right) .
$$

In this compact packing, generally particles are located near the wall and the central part of the box is less populated. As $l_{x} / d$ decreases from 1.2 to 1.0 , the $n_{x}\left(x=l_{x}\right)$, that is, the density at the side wall, first increases and then decreases, showing a (local) maximum around $l_{x} / d \simeq 1$.1. This property of local density variation is in accord with the variation of pressure $p_{x}$ shown in Fig. $1\left(l_{y} / d=1.2\right)$. Thus we consider that the density at a wall, which is determined through complicated packing of hard disks in a small rectangular box, is the main factor for the van der Waals behavior of $p_{x}$ in Fig. 1. In contrast to $n_{x}\left(x=l_{x}\right), n_{y}\left(y=l_{y}\right)$ turned out to increase monotonically as $l_{x} / d$ is decreased from 1.2 to 1 for $l_{y} / d$ $=1.2$. It is noted that whenever we observed the van der 


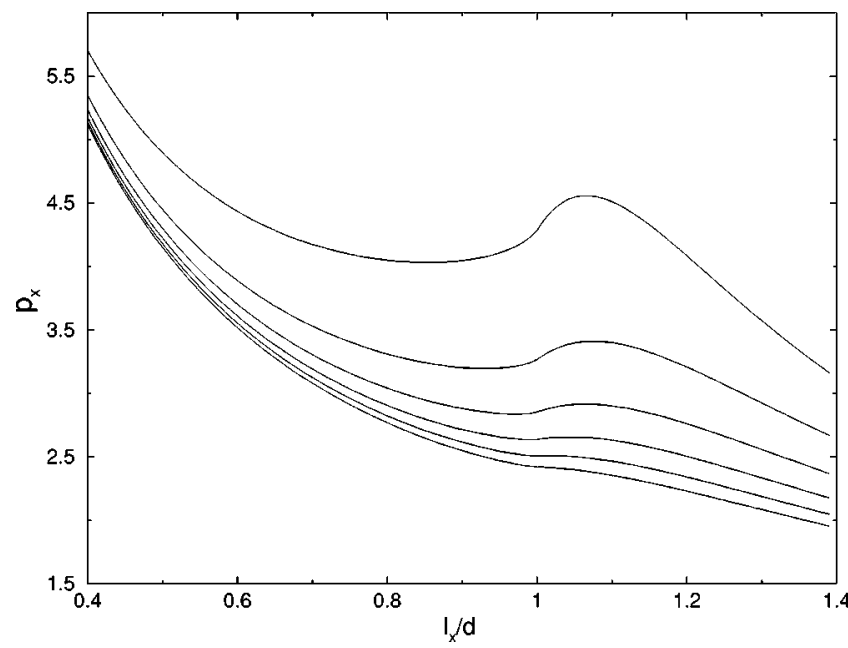

FIG. 1. Pressure on the vertical wall $p_{x}\left(l_{x}, l_{y}\right)$ for various $l_{y}$ values as functions of $l_{x}$. From the upper curve, $l_{y} / d=1.2,1.4,1.6$, 1.8, 2.0, and 2.2. Temperature $T$ and the Boltzmann constant are both set equal to one and the pressure is nondimensional.

Waals behavior we simultaneously observed the similar nonmonotonic behavior of particle density at the wall.

Qualitatively we may understand this van der Waals behavior as follows: First let us express $p_{x}$ as

$$
p_{x}=(1-W) p_{n-c o n}+W p_{c o n},
$$

where $W$ denotes the probability of two particles being in contact with each other along the $x$ axis, i.e., $\left|x_{2}-x_{1}\right|$ is around $d$, and $p_{c o n}$ the pressure in this situation which is larger than $p_{n \text {-con }}$ for the noncontact case. As $l_{x}$ becomes small from $l_{x} \gg d, p_{x}$ increases due to the increase of both $p_{n-c o n}$ and $p_{c o n}$. Under further compression, $p_{x}$ decreases first due to drastic decrease of $W$ and $W p_{\text {con }}$ and then increases due to increase of $p_{n-c o n}(W \simeq 0)$, resulting in the

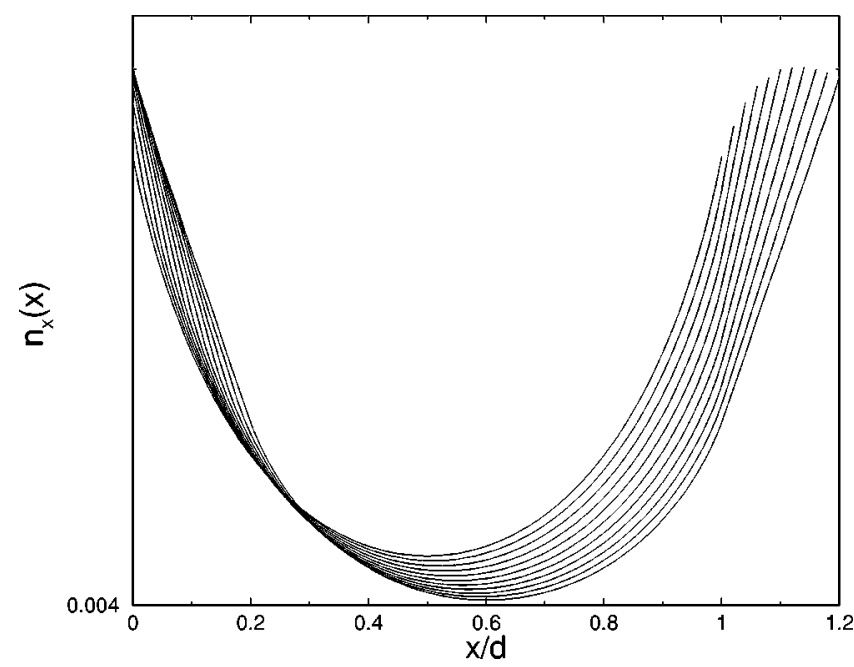

FIG. 2. Density profile $n_{x}(x)$ for $l_{y} / d=1.2$ and $l_{x}$ in the range between 1.0 and 1.2 with the increment $\Delta l_{x}=0.02$. It is noted that $x$ is in the range $0<x<l_{x}$.

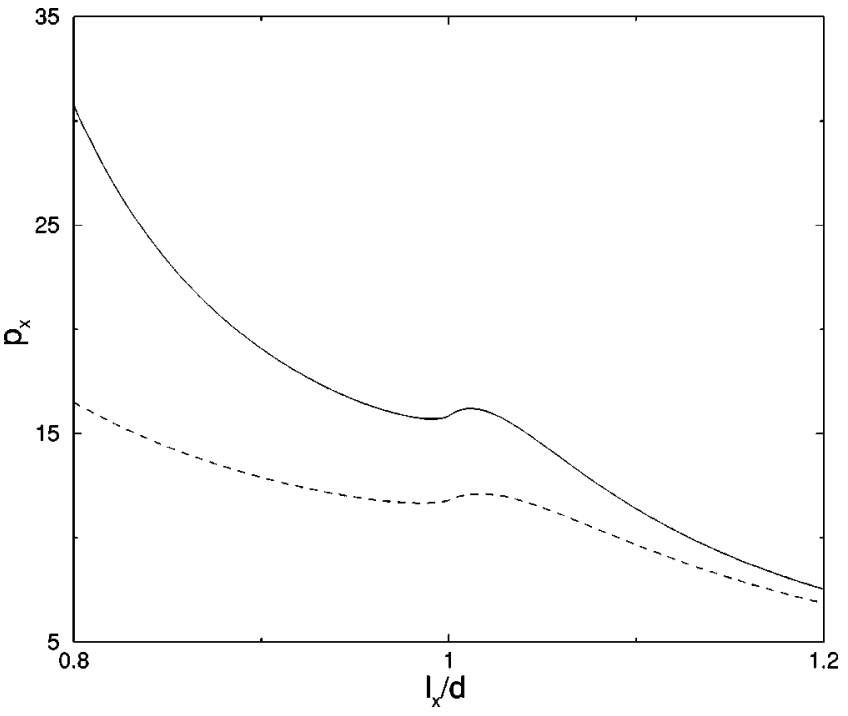

FIG. 3. $l_{x}$ dependence of $p_{x}$ for $l_{y} / d=0.75$ (full curve) and $l_{y} / d=0.85$ (dashed curve).

van der Waals behavior. A similar argument, if applied to the $l_{x}$ dependence of $p_{y}$, can explain the monotonic behavior mentioned above.

We now proceed to the region $l_{y}<d$, which was not investigated before. Just as for the case $l_{y}>d$, we observed the van der Waals behavior for $p_{x}\left(l_{x}, l_{y}\right)$ as shown in Fig. 3. This is also qualitatively explained based on Eq. (15). That is, under a closely packed situation, the van der Waals behavior may result from the change in packing mechanism, which accompanies the ergode-nonergode transition. This is in sharp contrast with the many-body hard-core system for which there is no distinction between a gas and a liquid. In the region $l_{y}<d$, there is an unstable region, in which the compressibility from $p_{x}$ becomes negative (see Fig. 3). As for $p_{y}$, we only observed a monotonous gaslike behavior for variation in $l_{x}$.

Collecting the results presented above for $p_{x}\left(l_{x}, l_{y}\right)$, we show in Fig. 4 the phase diagram in the $\left(l_{x}, l_{y}\right)$ plane. The

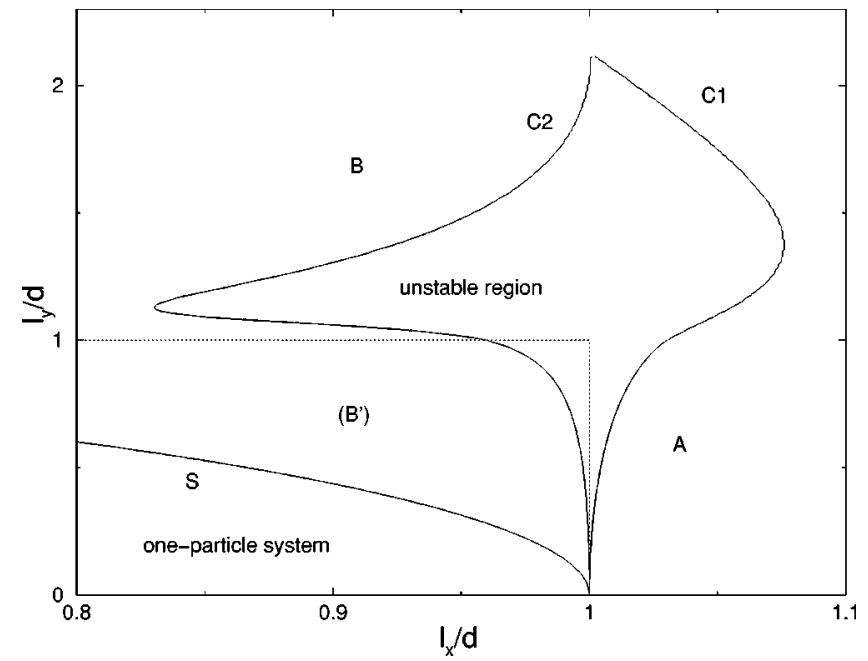

FIG. 4. Phase diagram in the $\left(l_{x}, l_{y}\right)$ space. 
two curves $C 1, C 2$ from $\left(d, l_{y, c}\right)$ to $(d, 0)$ determine the unstable region, in which the compressibility becomes negative. One may call the region $A$, which is either to the right of $C 1$ or above the line $l_{y}=l_{y, c}$, a gas phase and the region $B$, which is left to $C 2$, below the line $l_{y}=l_{y, c}$ and above $S$, a liquid phase. Inside the curve $S=\left(l_{x}, l_{y}\right): l_{x}^{2}+l_{y}^{2}=d^{2}$, the system can contain only one particle. However in region $B$, if we distinguish a fully constrained region $B^{\prime}: l_{x}<d, l_{y}<d$ from the remaining half-constrained region $l_{y}>d$ in $B$, one may call the system in $B^{\prime}$ a solid phase and the transition below the line $l_{y}<d$ becomes the gas solid one. We note that this phase diagram comes from the system response, more explicitly, the response of $p_{x}$ to $l_{x}$ variation, $p_{y}$ does not show any instability. If we study the response to $l_{y}$ variation, then $p_{y}$ shows instability and we only need to change the coordinates from the symmetry mentioned just below Eq. (14).

Finally, we comment on the probability distribution functions $f(x)$ and $g(y)$ for the relative positions. As a situation interesting from the viewpoint of application of the analytic expression for these functions, we consider that $l_{y} / d$ is larger than 1 and $l_{x} / d$ becomes gradually small and approaches 1 .
In order for the particle located in the upper part to exchange positions with the one in the lower part, the transition state, $y \equiv y_{2}-y_{1}=0$ must be crossed. If we put $l_{x} / d=1+\epsilon$ with $0<\epsilon \ll 1$, it is readily obtained from Eqs. (1) and (4) that $g(y=0)$ approaches $\epsilon^{2}$, showing that it takes a long time of the order $\epsilon^{-2}$ for two particles to exchange their relative (upper-lower) positions and this results in the slow relaxation observed in Ref. [5].

In this paper we considered a system composed of two hard disks based on analytic expressions for the partition function and some probability distribution functions. A system of two particles, which shows ideal or low-density gas properties if put in a box with large volume or under the periodic boundary condition, behaves quite differently in a compressed situation and shows a gas-liquid or a liquidsolid-like transition. Packing is one of the most important factors controlling properties of dense liquids [8] and amorphous substances (glass) $[9,10]$. Thus, we believe that investigation of properties of a few-body system packed in a small box might give important insights on condensed matter physics. Along this line some properties of a three-disk system and a thin two-dimensional $\mathrm{N}$-particle system will be dicussed in the near future.
[1] E. Ott, Chaos in Dynamical Systems (Cambridge University Press, Cambridge, 1993).

[2] L. E. Reichl, The Transition to Chaos (Springer-Verlag, Berlin, 1992).

[3] Z. Zheng, G. Hu, and J. Zhang, Phys. Rev. E 53, 3246 (1996).

[4] Gang Hu, Zhigang Zheng, Li Yang, and Wei Kang, Phys. Rev. E 64, 045102(R) (2001).

[5] A. Awazu, Phys. Rev. E 63, 032102 (2001).

[6] This is best called a gas-liquid transition for reasons to be clarified later.
[7] L. E. Reichl, A Modern Course in Statistical Physics (University of Texas Press, Austin, 1980).

[8] J. P. Hansen and I. R. MacDonald, Theory of Simple Liquids (Academic Press, New York, 1986).

[9] Liquids, Freezing and the Glass Transition, edited by J. P. Hansen, D. Levesque, and J. Zinn-Justin ( North-Holland, Amsterdam, 1989).

[10] E. Donth, The Glass Transition (Springer-Verlag, Berlin, 2001). 